\title{
Non-linear Economic Model Predictive Control of Water Distribution Networks
}

\author{
Ye Wang, ${ }^{\mathrm{a}, *}$ Vicenç Puig ${ }^{\mathrm{a}}$, Gabriela Cembrano ${ }^{\mathrm{a}, \mathrm{b}}$ \\ ${ }^{a}$ Advanced Control Systems (SAC) Research Group at Institut de Robòtica i Informàtica Industrial, \\ CSIC-UPC, Automatic Control Department, Universitat Politècnica de Catalunya-BarcelonaTech (UPC), \\ C/. Llorens i Artigas 4-6, 08028 Barcelona, Spain \\ ${ }^{b}$ Cetaqua, Water Technology Centre, Ctra. d'Esplugues 75, Cornellà de Llobregat, 08940 Barcelona, Spain
}

\begin{abstract}
This paper addresses a non-linear economic model predictive control (EMPC) strategy for water distribution networks (WDNs). A WDN could be considered as a non-linear system described by differential-algebraic equations (DAEs) when flow and hydraulic head equations are considered. As in other process industries, the main operational goal of WDNs is the minimisation of the economic costs associated to pumping and water treatment, while guaranteeing water supply with required flows and pressures at all the control/demand nodes in the network. Other operational goals related to safety and reliability are usually sought. From a control point of view, EMPC is a suitable control strategy for WDNs since the optimal operation of the network can not be established a priori by fixing reference volumes in the tanks. Alternatively, the EMPC strategy should determine the optimal filling/emptying sequence of the tanks taking into account that electricity price varies between day and night and that the demand also follows a 24-hour repetitive pattern. On the other hand, as a result of the $\mathrm{ON} / \mathrm{OFF}$ operation of parallel pumps in pumping stations, a two-layer control scheme has been used: a non-linear EMPC strategy with hourly control interval is chosen in the upper layer and a pump scheduling approach with one-minute sampling time in the lower layer. Finally, closed-loop simulation results of applying the proposed control strategy to the D-Town water network are shown.
\end{abstract}

Keywords: Economic model predictive control, pump scheduling approach, two-layer control scheme, non-linear differential-algebraic equations, water distribution networks

\section{Introduction}

Water distribution networks (WDNs) are critical infrastructures in urban areas. Their operational management is a subject of increasing interest, taking into account the economic

\footnotetext{
${ }^{*}$ Corresponding author.

Email addresses: ywang@iri.upc.edu (Ye Wang), vicenc.puig@upc.edu (Vicenç Puig), cembrano@iri.upc.edu (Gabriela Cembrano)
} 
and environmental factors. WDNs are also of vital importance for supporting all kinds of social activities. For the individuals living in a modern city, requiring sustainable water supply service is one of basic necessities. Besides, as the progress of society and the evolution of human civilisations, a growing number of people migrate into cities. Therefore, the increasing complexity of the WDN would cause some difficulties for the management under multiple objectives, such as economic operations, guaranteeing the whole system safe and stable.

Model predictive control (MPC) has attracted much attention of the research community all over the world throughout the past decades and has been applied to many different applications of the process industry [1,2]. Compared with the other classical control theories, the success is due to the fact that MPC is able to handle multi-input and multi-output (MIMO) control systems with hard and soft constraints for the system inputs and states, and meanwhile, having the ability to directly reach some certain system performances and operational objectives. In general, the MPC strategy (also regarded as receding horizon strategy) is based on finding the optimal control action from a sequence of open-loop control actions along the prediction horizon minimising a set of control objectives and satisfying a set of constraints including the system dynamic model and physical/operational limitations.

By investigating optimal control strategies for the management of water systems, MPC is not used in a classical way since there is no reference to be tracked. In applications of conventional MPC, the control objective is mainly focused on tracking a given reference or a family of trajectories in order to operate the plant to reach its steady state. Unlike conventional MPC, the common operational goal of many process industries, as WDNs, is the minimisation of economic costs of the energy consumptions. This lead to the so-called economic MPC (EMPC). The optimisation problem behind the EMPC strategy is in charge of finding a family of the optimal set-points taking into account economic benefits instead of driving the controlled system to a given set-point. The stability and cost improvement of EMPC have been investigated for non-linear processes in [3, 4], where Lyapunov-based technique has been employed over the conventional MPC. In order to reduce the computational complexity, the general EMPC is divided into two layers [5], where EMPC is set in the upper layer and a lower feedback control layer is used.

Related research for WDNs has been carried out in the past several years $[6,7,8,9,10$, $11,12,13,14,15]$. These research works are focused on finding the optimal operation on the WDN in order to achieve the desired control objectives. For this purpose, some optimal control problems are posed and solved to minimise the total operational costs of WDNs, for instance in $[6,16]$ the genetic algorithm (GA) is chosen. In $[7,8,10,13]$, authors have successfully applied the MPC strategy in the WDN using a control-oriented model that considers only flows, i.e., the pressure/head model of each element in the WDN including water storage tanks/reservoirs, water demand sectors, pressurised pipes, booster pumps and pressure/flow-controlled valves, are not considered explicitly. For a certain WDN, in addition to satisfy water demands, it is also necessary to meet the required pressure/head at each water demand sector and particular control points. A first attempt to consider the pressure/head model in the flow-based MPC is presented in [17], where the non-linear constraints coming from the flow-head equations are used to update the operational constraints 
of tanks and actuators by solving a constraint satisfaction problem before the flow-based linear MPC problem is solved. Later on, an explicit iterative approach to implement non-linear constraint relaxation is proposed in [15]. The non-linear hydraulic equations in the WDN model are relaxed to be a sequence of linear approximating constraints. The underlying linear optimisation problem with the relaxed constraints can produce similar performance.

The control-oriented model of a WDN may be built by a series of linear and non-linear differential-algebraic equations (DAEs). Subsequently, the non-linear DAE model of a WDN is used as the prediction model in the EMPC controller design. The application of EMPC to WDNs present some difficulties because of the non-linear nature of the DAE model and the $\mathrm{ON} / \mathrm{OFF}$ operation of the pumps. Because of these features, the MPC problem for WDNs leads to solve a non-linear mixed-integer problem [7]. In order to avoid solving such a complex optimisation problem, a two-layer optimal control scheme is proposed in this paper as shown in Figure 1. The upper layer is in charge of finding the optimal hourly flow set-points for actuators (pumps and valves) using a non-linear EMPC (NEMPC) strategy and the demand 24-hour forecasts using an appropriate forecasting algorithm, such as [18, 19]. So far, the conventional non-linear MPC has been applied to water systems but the use of NEMPC has still not been proposed [20,21]. The lower layer is in charge of the pump scheduling, i.e., the translation of the continuous flow set-points determined in the upper layer into $\mathrm{ON} / \mathrm{OFF}$ set-points for pump operation. Pumps are typically operated in $\mathrm{ON} / \mathrm{OFF}$ discrete way to produce at each hourly time-step, the same water volume as the optimal strategy computed by the first layer within an hour corresponding to each MPC step. By means of the two-layer scheme, the non-linear and integer features of the EMPC optimisation problem is decoupled in two problems: one dealing with the non-linear behaviours of WDNs and the other with discrete operations of the pumps.

The main contribution of this paper is to propose a two-layer control scheme that combines the NEMPC strategy in the upper layer, and the pump scheduling approach in the lower layer. The NEMPC strategy is implemented by using the non-linear programming technique and the pump scheduling approach is realised by solving a local optimisation problem. The proposed two-layer control strategy is validated using a hydraulic simulator that emulates the real WDN behaviour. The D-Town water network, a well known benchmark, is used as the case study. The closed-loop simulation is implemented using a simulation platform with a virtual-reality hydraulic simulator that emulates the on-line operation.

The remainder of this paper is structured as follows: The control-oriented model of WDNs including the flow and pressure/head variables is presented in Section 2. The NEMPC strategy of the WDN in the upper layer is introduced in Section 3. A pump scheduling approach in the lower layer is provided in Section 4. The description and introduction of the case study of the D-Town water network is presented in the Section 5. In Section 6, results of applying the proposed control strategy to the D-Town water network are summarised. Finally, some conclusions are drawn in Section 7. 


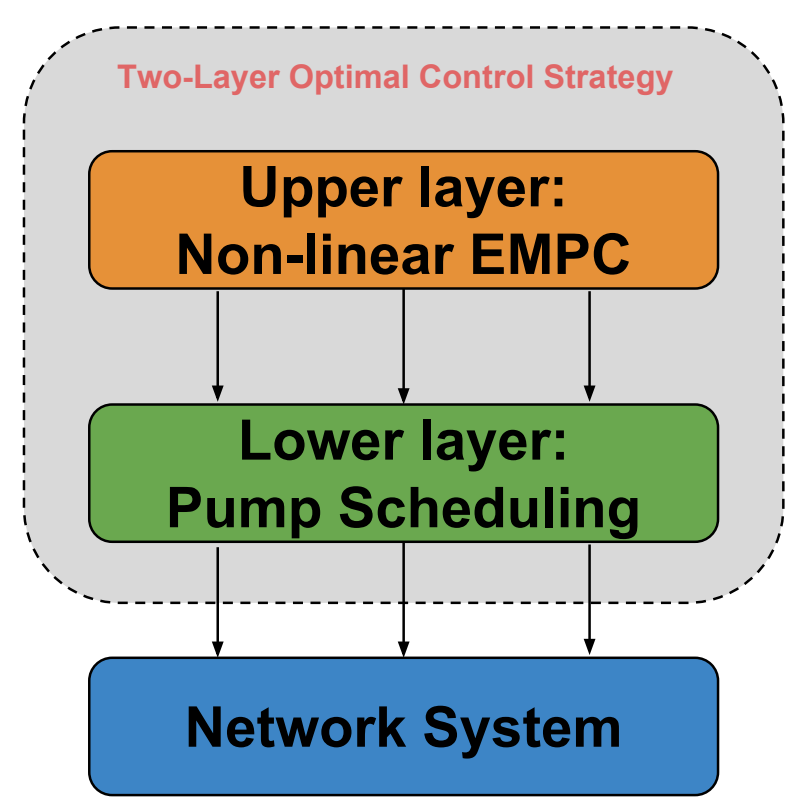

Figure 1: Two-layer optimal control strategy of WDNs

\section{Control-oriented modelling water distribution networks}

This section briefly introduces the control-oriented mathematical modelling methodology of the WDN including the flow and hydraulic head relations for the different network components. As result of the application of this methodology to a particular WDN, a set of dynamic and static relationships that lead to a system of DAEs in discrete-time ready to be used in the implementation of the MPC is obtained. A WDN can be decomposed by a set of constitutive elements: reservoirs/tanks, control valves, pump stations, nodes and water demand sectors, each being characterised by means of flow-head relations [8, 16, 22, 23].

\subsection{Tanks}

Water tanks supply and provide the entire WDN with the storage capacity of drinking water to consumers guaranteeing adequate water pressure service. The mass balance expression relating the stored volume $v$ in the $m$-th tank can be written as the discrete-time difference equation which describes the tank dynamical evolution as

$$
v_{m}(k+1)=v_{m}(k)+\Delta t\left(\sum_{i} q_{i, m}^{\mathrm{in}}(k)-\sum_{j} q_{m, j}^{\mathrm{out}}(k)\right),
$$

where $q_{i, m}^{\text {in }}(k)$ denotes the inflows from the $i$-th element to the $m$-th tank and $q_{m, j}^{\text {out }}(k)$ denotes the outflows from the $m$-th tank to the $j$-th element. $\Delta t$ is the sampling time and $k$ is the discrete-time instant. The physical limitation related to the storage volume in the $m$-th tank is described as

$$
\underline{v_{m}} \leq v_{m}(k) \leq \overline{v_{m}}, \quad \forall k \in \mathbb{N}_{+}
$$




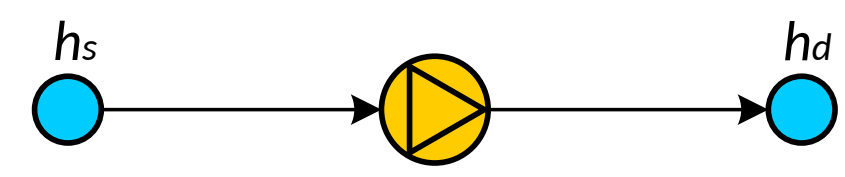

(a) Pump

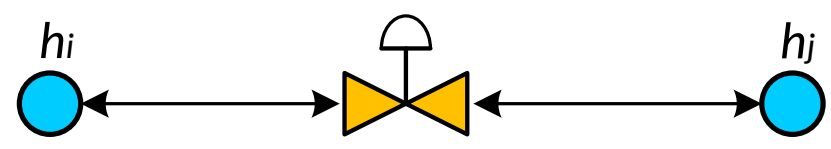

(b) Valve

Figure 2: Conceptual models of the pump and valve

where $v_{m}$ and $\overline{v_{m}}$ denote the minimum and maximum admissible storage capacity, respectively.

The head model in WDN is typically written in terms of the hydraulic head that relates the energy in an incompressible fluid to the height of an equivalent static column of that fluid. Note that head is expressed in units of height.

Using this concept, the head related to the $m$-th tank with respect to the volume of storage water inside can be determined as follows:

$$
h_{m}(k)=\frac{v_{m}(k)}{\mathrm{S}_{m}}+E_{m}, \quad \forall k \in \mathbb{N}_{+},
$$

where $\mathrm{S}_{m}$ is the cross-sectional area of the $m$-th tank and $E_{m}$ corresponds the $m$-th tank elevation.

\subsection{Pumping stations}

Pumps located in pumping stations of a WDN can be of several types: fixed-speed pumps (FSP), variable-speed pumps (VSP) and variable throttle pumps (VTP) [22] depending on how they are controlled. In this paper, we will consider fixed-speed pumps that are the most used in WDN because of the simplicity of operation, i.e., they are operated in an ON/OFF manner. However, such simplicity introduces an additional problem when implementing a continuous control strategy as MPC since the ON/OFF operation involves including discrete variables in the optimisation problem.

Pump flows are regarded as the manipulated variables. Therefore, the physical limitations for pumps can be regarded as input constraints, which can be expressed as

$$
\underline{q_{u_{n}}} \leq q_{u_{n}}(k) \leq \overline{q_{u_{n}}}, \quad \forall k \in \mathbb{N}_{+},
$$

where $q_{u_{n}}$ represents the manipulated flow of the $n$-th pump (or valve), $q_{u_{n}}$ and $\overline{q_{u_{n}}}$ represent the minimum and maximum flow capacity of the $n$-th pump, respectively. These limitations vary with the pressure according to hydraulic flow/head curve of the pump.

The hydraulic characteristic of a pump is formulated by a non-linear function related to the flow and head variables. Therefore, for a pump shown in Figure 2(a), the hydraulic characteristics are bounded by the following constraints: 


$$
\begin{aligned}
& \Delta h_{p}(k)=h_{d}(k)-h_{s}(k) \geq 0, \quad \forall k \in \mathbb{N}_{+}, \\
& h_{d}(k) \in\left[\underline{h_{d}}, \overline{h_{d}}\right], \\
& h_{s}(k) \in\left[\underline{h_{s}}, \overline{h_{s}}\right],
\end{aligned}
$$

where $h_{d}(k)$ and $h_{s}(k)$ denote the suction head and the delivery head at time instant $k$, respectively, with the physical limitation of $h_{d}(k) \geq h_{s}(k)$. Moreover, $\underline{h_{d}}$ and $\underline{h_{s}}$ denote the minimum values of the suction and delivery heads. $\overline{h_{d}}$ and $\overline{h_{s}}$ denote the maximum values of the suction and delivery heads.

\subsection{Valves}

In terms of the type of valves, there is a variety of options, such as pressure modulating, non-return, pressure reducing, flow variable control, head control and so on [22]. For simplicity, valves considered in this paper are of the flow-control type.

Similar to pumps, the characteristic of valves is difficult to be modelled. Hence, the static relationship between head and flow of a valve shown in Figure 2(b) is constrained as follows:

$$
\begin{aligned}
& \Delta h_{v}(k)=h_{u s}(k)-h_{d s}(k) \geq 0, \quad \forall k \in \mathbb{N}_{+}, \\
& h_{u s}(k) \in\left[\underline{h_{u s}}, \overline{h_{u s}}\right], \\
& h_{d s}(k) \in\left[\underline{h_{d s}}, \overline{h_{d s}}\right],
\end{aligned}
$$

where $h_{u s}(k)$ and $h_{d s}(k)$ denote the heads at the nodes around the valve in the upstream and downstream at time instant $k$, respectively. $\underline{h}_{u s}$ and $\underline{h_{d s}}$ denote the minimum values of the upstream and downstream heads. $\overline{h_{u s}}$ and $\overline{h_{d s}}$ denote the maximum values of the upstream and downstream heads.

\subsection{Nodes}

Water flow through each node of the network must fulfil the mass balance relations. The expression of the mass conservation in these nodes can be written as

$$
\sum_{i} q_{i, l}^{\text {in }}(k)=\sum_{j} q_{l, j}^{\text {out }}(k), \quad \forall k \in \mathbb{N}_{+},
$$

where $q_{i, l}^{\text {in }}$ represents the non-manipulated inflow through $l$-th node from the $i$-th element and $q_{l, j}^{\text {out }}$ represents the non-manipulated outflow through $l$-th node to the $j$-th element.

\subsection{Water demand sectors}

A demand sector represents water demand of the network users of a certain physical area. At a certain time instant $k$, the consumed water in the $r$-th demand sector can be expressed as $d_{r}(k)$. Since the optimal control strategy is considered as a predictive one, the short-term demand forecasts are able to obtain by using a suitable demand forecasting algorithm, such as $[18,19]$. 
Pipes convey water from one place in the network to another. Water inside pressurised pipes flows from the higher hydraulic head to that at lower head. Therefore, the head-flow relationship for a pipe can be described as

$$
q_{i, j}(k)=\Phi_{i, j}\left(h_{i}(k)-h_{j}(k)\right),
$$

where $\Phi_{i, j}$ is a non-linear relationship, usually described by an empirical equation, for instance, the Hazen-Williams formula. Hence, the head drop through a pipe $\Delta h_{d}(k)$ for $\forall k \in \mathbb{N}_{+}$can be calculated as

$$
\Delta h_{d}(k)=h_{i}(k)-h_{j}(k)=R_{i, j} q_{i, j}(k)\left|q_{i, j}(k)\right|^{0.852},
$$

with

$$
R_{i, j} \triangleq \frac{10.67 L_{i, j}}{C_{i, j}^{1.852} D_{i, j}^{4.87}}
$$

where $L_{i, j}, D_{i, j}$ and $C_{i, j}$ denote the pipe length, diameter and roughness coefficient, respectively.

Basically, pipes can be classified based on the flow sense into unidirectional and bidirectional. Therefore, $\Delta h_{d}(k)$ for $\forall k \in \mathbb{N}_{+}$in the unidirectional pipe is always positive with its selected direction while in the bidirectional pipe $\Delta h_{d}(k)$ for $\forall k \in \mathbb{N}_{+}$could be varying between positive and negative since the direction of the flow can be reversed.

\section{Non-linear economic model predictive control of water distribution networks}

\subsection{Control-oriented model of the water distribution network}

Considering the modelling methodology of each component in the WDN presented above, the control-oriented model of WDNs can be formulated by means of DAEs. The generalised discrete-time DAE model can be written as follows:

$$
\begin{aligned}
x(k+1) & =F(x(k), z(k), u(k), w(k), d(k)), \\
0 & =G(x(k), z(k), u(k), w(k), d(k)),
\end{aligned}
$$

where $x \in X \subseteq \mathbb{R}^{m}$ represents the vector of hydraulic heads at storage nodes (tanks) as differential states, $z \in Z \subseteq \mathbb{R}^{z}$ represents the vector of hydraulic heads at non-storage nodes as algebraic states, $u \in U \subseteq \mathbb{R}^{n}$ denotes the vector of the manipulated flows through actuators (pumps and valves) as control inputs, $w \in W \subseteq \mathbb{R}^{w}$ denotes the vector of nonmanipulated flows through interconnected pipes and $d \in D \subseteq \mathbb{R}^{d}$ corresponds to the vector of water demands as system disturbances. $k \in \mathbb{N}_{+}$denotes the time instant. $F(\cdot)$ and $G(\cdot)$ are vectors of mapping functions. Moreover, (10a) is the discrete-time differential equation describing the system dynamics while (10b) is the discrete-time algebraic equation presenting 
the static relations of components in the WDN. All the considered variables of the WDN in Section 2 are classified as control-oriented variables as summarised in Table 1. Considering that the tanks are the only elements with dynamics in the WDN, (10a) can be explicitly expressed as

$$
x(k+1)=A x(k)+B_{u} u(k)+B_{w} w(k)+B_{d} d(k),
$$

where $A, B_{u}, B_{w}$ and $B_{d}$ are system matrices of appropriate dimensions.

Table 1: Variable assignments in the control-oriented model of the WDN

\begin{tabular}{|c|c|c|}
\hline Type of variable & Related symbols & Description \\
\hline Difference states: $x$ & $h_{m}$ & $\begin{array}{l}\text { Hydraulic heads at the storage } \\
\text { nodes (i.e. storage tanks) }\end{array}$ \\
\hline Algebraic states: $z$ & $h_{d}, h_{s}, h_{i}, h_{j}$ & $\begin{array}{l}\text { Hydraulic heads at the non- } \\
\text { storage nodes }\end{array}$ \\
\hline Control inputs: $u$ & $q_{u_{n}}$ & $\begin{array}{l}\text { Manipulated flows through actu- } \\
\text { ators (pumps and valves) }\end{array}$ \\
\hline Non-control inputs: $w$ & $q_{i, j}$ & $\begin{array}{l}\text { Non-manipulated flows through } \\
\text { interconnected pipes }\end{array}$ \\
\hline System disturbances: $d$ & $d_{r}$ & Water demands \\
\hline
\end{tabular}

Furthermore, considering the static relations related to flow and head variables in (3), (7) and (9), the static equation (10b) can be explicitly reformulated as follows:

$$
\begin{aligned}
& 0=E_{u} u(k)+E_{w} w(k)+E_{d} d(k), \\
& 0=P_{x} x(k)+P_{z} z(k)+\psi(w(k)),
\end{aligned}
$$

where (12a) describes the mass balance equations of flow variables at non-storage nodes in the WDN and the dynamic of $z(k)$ is related to $x(k)$ in $(12 \mathrm{~b})$. Besides, $E_{u}, E_{w}, E_{d}, P_{x}$ and $P_{z}$ are system matrices of appropriate dimensions determined by the network topology. Moreover, $\psi(\cdot)$ denotes the vector of non-linear Hazen-Williams mapping functions.

In general, the control-oriented model of the WDN can be written as

$$
\begin{aligned}
x(k+1) & =A x(k)+B_{u} u(k)+B_{w} w(k)+B_{d} d(k), \\
0 & =E_{u} u(k)+E_{w} w(k)+E_{d} d(k), \\
0 & =P_{x} x(k)+P_{z} z(k)+\psi(w(k)) .
\end{aligned}
$$

Note that units of all the control-oriented variables need to be consistent. In this paper, the unit of the head is selected as $m$ (meter). The water flows is with unit of $\mathrm{m}^{3} / \mathrm{s}$ (cubicmeter per second). 


\subsection{Cost function settings}

\subsubsection{Management criteria of the water distribution network}

The operational goals for the management of the WDN include:

- Economic: To provide a reliable water supply with the required pressure minimising operational costs;

- Safety: To guarantee the availability of enough water with suitable pressure in each storage tank to satisfy its underlying uncertain water demands;

- Smoothness: To operate actuators (pumps and valves) in the WDN under smooth control actions.

\subsubsection{Minimisation of water distribution costs}

The main control objective of the WDN is to minimise the water distribution costs that includes water acquisition costs and electrical costs especially for pumping water through the pumps. The water is delivered into the nodes with different heads (and elevations) through the distribution network implying many electrical costs on the booster pumping. Therefore, the cost function associated to this objective can be formulated as

$$
\ell_{1}(k) \triangleq\left(\alpha_{1}+\alpha_{2}(k)\right)^{T} u(k),
$$

where $\alpha_{1}$ denotes the single-column vector of static economic costs of the water depending on the selected water sources and $\alpha_{2}(k)$ represents the vector of the time-varying electrical costs. Considering the variable daily electrical tariff, $\alpha_{2}(k)$ is time-varying.

\subsubsection{Guarantee of safe water storage}

For the purpose of maintaining the water supply in spite of the variation of water demands between two consecutive MPC sampling steps, a suitable safety head for each storage tank is necessary to be maintained. Hence, the mathematical expression for this objective is formulated in a quadratic way as

$$
\ell_{2}(k) \triangleq \begin{cases}\left\|x(k)-x_{S}\right\|_{2}^{2}, & \text { if } x(k) \leq x_{S}, \\ 0, & \text { otherwise, }\end{cases}
$$

where $x_{S}$ denotes the vector of the safety heads for all the tanks and $\|\cdot\|_{2}^{2}$ is the squared 2-norm symbol. This cost function can be also realised by means of a soft constraint with adding a slack variable $\xi(k)$, which can be reformulated as

$$
\ell_{2}(k) \triangleq\|\xi(k)\|_{2}^{2},
$$

in addition to the following soft constraint:

$$
x(k) \geq x_{S}-\xi(k) .
$$




\subsubsection{Smoothness of control actions}

The actuators in the WDN mainly includes pumps and valves. Thus, the flow-based control actions found by the EMPC controller is required to be smooth in order to maximise the lifespan of the actuators. In addition, the use of the smooth operations is potentially benefit for the lower-layer regulatory performance. To achieve a sequence of smooth operations, the slew rate of the control actions between two consecutive time steps is penalised. Hence, the cost function for this part can be written as

$$
\ell_{3}(k) \triangleq\|\Delta u(k)\|_{2}^{2}
$$

where

$$
\Delta u(k) \triangleq u(k)-u(k-1)
$$

\subsubsection{Multi-objective cost function}

In general, the multi-objective cost function that gathers all the control objectives for the operational management of the WDN can be summarised as

$$
\mathcal{J}(k)=\sum_{j=1}^{\Gamma} \lambda_{j} \ell_{j}(k),
$$

where $\lambda_{j}$ denotes the weighting term that indicates the prioritisation of control objectives and $\Gamma$ is the number of the selected control objectives.

\subsection{System constraint settings}

In the real components of a WDN, there are the physical limitations associated to the system variables. Therefore, these constraints should complement the mass balance principles and physical relations between flow and head introduced in (13). In the following, these physical constraints are described in detail.

\subsubsection{Tank capacity constraints}

The hard constraint on the system states $x$ comes from the tank capacity in the WDN, which can be described as

$$
\underline{x_{i}} \leq x_{i}(k) \leq \overline{x_{i}}, \quad \forall k \in \mathbb{N}_{+} \text {and } i \in[1, m] \subset \mathbb{Z}_{+},
$$

where $x_{i}$ and $\overline{x_{i}}$ represent the minimum and maximum heads with respect to capacities of the $i$-th tank, respectively. The tank volumetric capacity can be transformed into hydraulic head constraints by $(3)$. 


\subsubsection{Flow constraints on actuators}

The manipulated flows are also limited taking the physical capacity of different actuators, which can be expressed as

$$
\underline{u_{i}} \leq u_{i}(k) \leq \overline{u_{i}}, \quad \forall k \in \mathbb{N}_{+} \text {and } i \in[1, n] \subset \mathbb{Z}_{+},
$$

where $u_{i}$ and $\overline{u_{i}}$ denote the minimum and maximum manipulated flows of the $i$-th actuator, respectively. On the other hand, the non-manipulated flows throughout the interconnected pipes can be limited between $\underline{w_{i}}$ and $\overline{w_{i}}$ as

$$
\underline{w_{i}} \leq w_{i}(k) \leq \overline{w_{i}}, \quad \forall k \in \mathbb{N}_{+} \text {and } i \in\left[1, n_{p}\right] \subset \mathbb{Z}_{+},
$$

where $n_{p}$ is the number of pipes.

\subsubsection{Head constraints on demand sectors}

The heads at some certain non-storage nodes are required to be up to some minimum levels as in the case of the water demand sectors. Hence, the following inequality constraint is necessary to be considered:

$$
z_{i}(k) \geq \underline{z_{i}}, \quad \forall k \in \mathbb{N}_{+} \text {and } i \in\left[1, n_{h}\right] \subset \mathbb{Z}_{+},
$$

where $z_{i}$ are the required heads at the water demand sectors. Moreover, $n_{h}$ is the total number of the water demand sectors.

\subsection{NEMPC optimisation problem formulation}

In general, the NEMPC strategy can be implemented by solving a finite-horizon optimisation problem over a prediction horizon $H_{p}$, where the multi-objective cost function is minimised subject to the prediction model and a set of system constraints. Thus, the optimisation problem behind the NEMPC strategy can be formulated as follows:

\section{Problem 1 (NEMPC of the WDN).}

$$
\min _{u(k \mid k), \ldots, u\left(k+H_{p}-1 \mid k\right)} \sum_{i=0}^{H_{p}-1} \sum_{j=1}^{\Gamma} \lambda_{j} \ell_{j}(k+i \mid k),
$$

subject to

$$
\begin{aligned}
& x(k+i+1 \mid k)=A x(k+i \mid k)+B_{u} u(k+i \mid k)+B_{w} w(k+i \mid k)+B_{d} d(k+i \mid k), \\
& 0=E_{u} u(k+i \mid k)+E_{w} w(k+i \mid k)+E_{d} d(k+i \mid k), \\
& 0=P_{x} x(k+i \mid k)+P_{z} z(k+i \mid k)+\psi(w(k+i \mid k)), \\
& \underline{x} \leq x(k+i+1 \mid k) \leq \bar{x}, \\
& \underline{u} \leq u(k+i \mid k) \leq \bar{u}, \\
& z(k+i \mid k) \geq \underline{z} \\
& x(k+i+1 \mid k) \geq x_{S}-\xi(k+i), \\
& (x(k \mid k), d(k \mid k))=(x(k), d(k)) .
\end{aligned}
$$


Since the control-oriented model of the WDN includes the non-linear relations in (25d), the above optimisation problem naturally becomes non-linear. Thus, Problem 1 should be solved using a suitable non-linear programming technique. Assuming that Problem 1 is feasible, the sequence of control actions can be given as

$$
u^{*}(k)=\left[u^{*}(k \mid k), \quad u^{*}(k+1 \mid k), \quad \cdots, u^{*}\left(k+H_{p}-1 \mid k\right)\right] .
$$

And then by deploying the receding-horizon strategy, the optimal control action at time instant $k$ is the first component of the sequence of control actions denoted by

$$
u_{\text {opt }}(k) \triangleq u^{*}(k \mid k)
$$

\section{Pump scheduling approach}

In practice, the main energy consumption is used for pumping water through the pumping stations. In case of the pumps with ON/OFF operation, the flows in (26) become discrete values and subsequently Problem 1 becomes a non-linear mixed-integer problem. In this section, this non-linear mixed-integer optimisation problem is firstly introduced. In order to avoid solving this complicated optimisation problem, a two-layer control strategy is proposed including the NEMPC strategy and the pump scheduling approach.

\subsection{Pump efficiency in ON/OFF operation}

A pump operated at a fixed speed in an ON/OFF manner delivers a flow between zero and a maximum value that depends of boundary head drops conditions. Commonly, the pump performance is typically characterised by the flow-head and efficiency curves as shown in Figure 3. Figure 3(a) presents the relationship between hydraulic head and the delivered flow through the considered pump. Figure 3(b) presents the operational efficiency of the pump in a function of the water flow through the pump. From these two figures, it can be observed that flow and head present an inverse relation, that is, if the flow increases the water head provided by the pump decreases. On the other hand, the maximum pump efficiency is obtained for a given flow that can be determined by the efficiency curve. Thus, when selecting a pump, these curves should be taken into account in such a manner the pump operates in the optimal efficiency region.

The cost $\alpha_{2}(k)$ in (14) can be calculated taking into account the electricity price from a given tariff and pump efficiency at time instant $k$ as [24]

$$
\alpha_{2}(k)=\frac{\rho_{w} \cdot g \cdot \Delta z(k) \cdot \varphi_{e}(k)}{\eta(k)},
$$

where $\rho_{w}$ is the water density (approximately $1000 \mathrm{~kg} / \mathrm{m}^{3}$ ), $g$ is the acceleration corresponding to the gravity $\left(9.8 \mathrm{~m} / \mathrm{s}^{2}\right), \varphi_{e}(k)$ denotes the electricity price per kilowatt-hour at the time instant $k$ with unit of $(€ / k W h), \Delta z(k) \triangleq z_{i}(k)-z_{j}(k)$ denotes the gained head for the 


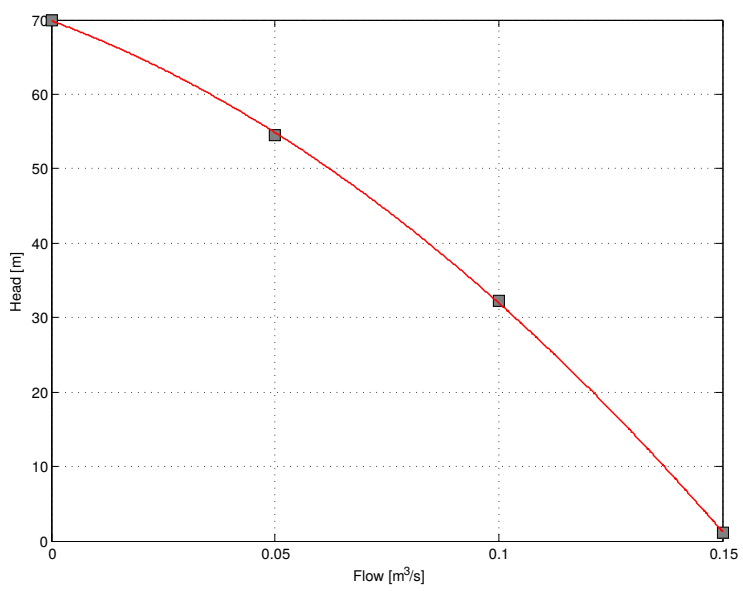

(a) Pumping curve

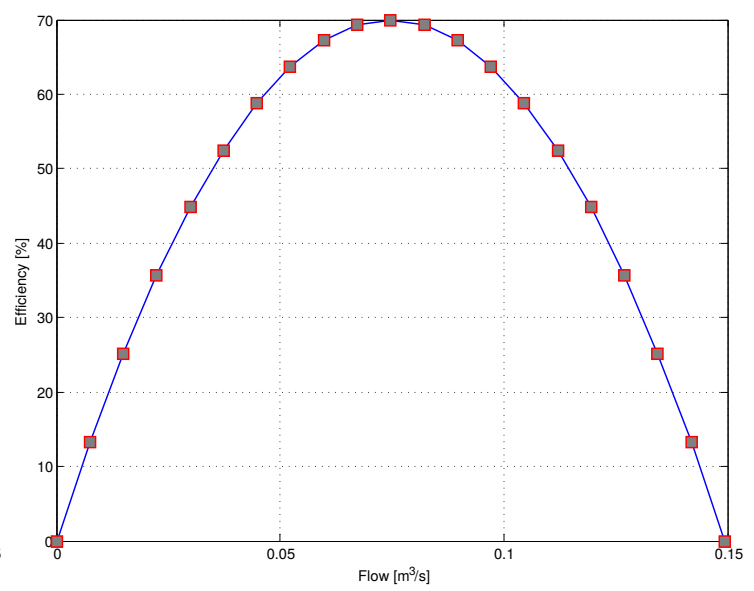

(b) Efficiency curve

Figure 3: Pump characteristic curves

pumping water $(m)$ and $\eta(k)$ represents the pump wire-to-water efficiency ${ }^{1}(\%)$ due to the flow at time instant $k$. The efficiency curves can be built by approximating the polynomial functions with experimental data or provided by the pump manufacturer.

\subsection{Mixed-integer NEMPC including discrete ON/OFF pump scheduling}

Considering that pumps are operated in an ON/OFF discrete manner, Problem 1 should be reformulated. By introducing new time-varying binary decision variable $\zeta(k) \in$ $\{0,1\}$ that correspond to the ON/OFF operation of the pumps, the pumping flow $u(k)$ at time instant $k$ should be replaced by

$$
u(k)=\zeta(k) \tilde{u}(k),
$$

where the decision variable $u(k)$ involves a binary variable $\zeta(k)$ representing ON/OFF pump status and $\tilde{u}(k)$ corresponding to the flow when the pump is ON. Therefore, the cost function (1) can be rewritten considering (29) as $\tilde{\ell}_{j}(\cdot)$ for $j \in[1, \Gamma] \subset \mathbb{Z}_{+}$.

Thus, Problem 1 is reformulated as a mixed-integer optimisation problem in the following:

\section{Problem 2 (Mixed-integer NEMPC of the WDN).}

$$
\min _{\substack{\zeta(k), \ldots, \zeta\left(k+H_{p}-1 \mid k\right) \\ \tilde{u}(k \mid k), \ldots, \tilde{u}\left(k+H_{p}-1 \mid k\right)}} \sum_{i=0}^{H_{p}-1} \sum_{j=1}^{\Gamma} \lambda_{j} \tilde{\ell}_{j}(k+i \mid k),
$$

\footnotetext{
${ }^{1}$ Pump Efficiency indicates the percentage of brake horsepower converted into useful work. Pump efficiency, along with flow, head, and liquid specific gravity affect the power required to drive the pump. The more efficient pump, the less power required to drive it.
} 
subject to

$$
\begin{aligned}
& x(k+i+1 \mid k)=A x(k+i \mid k)+B_{u} \zeta(k+i) \tilde{u}(k+i \mid k)+B_{w} w(k+i \mid k)+B_{d} d(k+i \mid k), \\
& 0=E_{u} \zeta(k+i) \tilde{u}(k+i \mid k)+E_{w} w(k+i \mid k)+E_{d} d(k+i \mid k), \\
& 0=P_{x} x(k+i \mid k)+P_{z} z(k+i \mid k)+\psi(w(k+i \mid k)), \\
& \underline{x} \leq x(k+i+1 \mid k) \leq \bar{x} \\
& \underline{u} \leq \zeta(k+i) \tilde{u}(k+i \mid k) \leq \bar{u} \\
& z(k+i \mid k) \geq \underline{z} \\
& x(k+i+1 \mid k) \geq x_{S}-\xi(k+i), \\
& \zeta(k+i) \in\{0,1\} \\
& (x(k \mid k), d(k \mid k))=(x(k), d(k)),
\end{aligned}
$$

where $\zeta(k+i) \in\{0,1\}$ represents the vector of binary decision variables that correspond to the $\mathrm{ON} / \mathrm{OFF}$ pump status at time instant $k$, where 0 and 1 corresponds to the ON-status and OFF-status of the pumps, respectively. The ON-status pumping flow $\tilde{u}(k)$ should be estimated based on the pressure conditions that the pumps should operate defined in (5). Problem 1 is considered to operate at hourly basis, which means that optimal hourly flows for actuators are determined. In Problem 2, the sampling time should be shorter (at the minutely scale) in order to approximate the optimal continuous flow that would be obtained by solving Problem 1 assuming that actuator flows are manipulated in a continuous manner.

Solving a large-scale non-linear mixed-integer optimisation problem with a long horizon leads Problem 1 to be computationally complex without a guaranteed convergence in a limited time, for the purpose of real-time control. For this reason, Problem 2 is proposed to be replaced by a two-layer optimal control strategy.

\subsection{Two-layer optimal control strategy}

\subsubsection{The upper layer: NEMPC strategy}

The two-layer optimal control strategy is presented by gathering all the considerations discussed before. In the upper layer, Problem 1 is adopted. Note that the NEMPC of the WDN is operated on the hourly basis $\left(\Delta t_{u}=3600 s\right)$ over the MPC prediction horizon $H_{p}$ to find the optimal flow set-points. In the lower layer, the sampling time is usually selected as one minute $\left(\Delta t_{l}=60 \mathrm{~s}\right)$, which means that the optimal flow set-points at one MPC step decides 60 values using the pump scheduling approach.

\subsubsection{The lower layer: pump scheduling approach}

Usually, the pumping stations are set independently in a WDN. Hence, the control objective in the lower layer is to find a set of ON-OFF sequences for each pumping station. Denote $Q_{j}^{o p t}=u_{o p t_{j}}$ for $\forall j \in\left[1, n_{s}\right] \subset \mathbb{Z}_{+}$as the optimal hourly flow set-point of the $j$-th pumping station obtained from the upper layer, where $n_{s}$ is the total number of pumping stations in a WDN.

The control objectives of the lower layers can be summarised as follows: 
- To provide enough water to reach the optimal water flow set-points.

- To use the minimum possible number of parallel pumps and avoid too many switches in order to maximise their working lives.

In terms of the $j$-th pumping station, the pumping flow of the $i$-th pump is affected by the factors of the suction and delivery heads. Hence, if these boundary heads are given, the actual flow $q_{i, j}^{r}$ through the pump is considered within an interval, which can be formulated as

$$
q_{i, j}^{r} \in\left[q_{i, j}^{n}-\sigma_{i, j}, q_{i, j}^{n}+\sigma_{i, j}\right],
$$

where $q_{i, j}^{n}$ denotes nominal pumping flow produced through the $i$-th pump, and $\sigma_{i, j}$ represents the variance of the pumping flow depending on the uncertainty of the boundary heads. It is assumed that the actual flow $q_{i, j}^{r}$ can be measured. In some cases, only one pump cannot provide enough flows to maintain the optimal flow set-point. Hence, parallel pumps are set in each pumping station. Ideally, the optimal pumping flow $Q_{j}^{o p t}$ can be satisfied when all the pumps are open in the lower layer such that the following condition holds:

$$
Q_{j}^{o p t} \Delta t_{u}=\sum_{i=1}^{n_{c_{j}}} \sum_{t=1}^{H_{l}} q_{i, j}^{r} \Delta t_{l} .
$$

where $n_{c_{j}}$ is the total number of parallel pumps in the $j$-th pumping station and $H_{l}$ is the control horizon of the lower layer.

Consider that the parallel pumps are operated in ON/OFF way, the binary variable $\chi_{i, j}(t) \in\{0,1\}$ at time instant $t$ is chosen, where $\chi_{i, j}(t)=0$ describes the OFF-status and $\chi_{i, j}(t)=1$ presents the ON-status. Therefore, the actual flow of the $i$-th pump can be computed by

$$
q_{i, j}(t)=\chi_{i, j}(t) q_{i, j}^{r}, \quad \forall i \in\left[1, n_{c_{j}}\right] \subset \mathbb{Z}_{+}, \forall t \in\left[1, n_{s}\right] \subset \mathbb{Z}_{+} .
$$

Furthermore, the minimum usages of required parallel pumps and switches are necessary to be taken into account. It is considered that the parallel pumps are selected in a sequence order from $i=1$ to $i=n_{c_{j}}$. Therefore, the required parallel pumps for $j$-th pumping station can be constrained by the following condition:

$$
\chi_{i+1, j}(t)+\left(1-\chi_{i, j}(t)\right) \leq 1, \quad \forall i \in\left[1, n_{c_{j}}\right] \subset \mathbb{Z}_{+},
$$

which means if $i$-th pump is not used, then $i+1$-th pump is also not used. Additionally to (34), the minimum required parallel pumps with their selection orders can be decided by maximising the following term:

$$
\mathcal{J}_{p}=\sum_{i=1}^{n_{c_{j}}} \sum_{t=1}^{H_{l}} \mu_{i} \chi_{i, j}(t)
$$


where $\mu_{i}>0$ for $\forall i \in\left[1, n_{c_{j}}\right] \subset \mathbb{Z}_{+}$. Considering the pump operations in a order, the lexicographic prioritisation is used to set this sequence of weights as $\mu_{1}>\cdots>\mu_{n_{c_{j}}}[11]$.

On the other hand, during total horizon of the lower layer, the used parallel pumps are switched once in order to get smooth control actions. The required pumps are used at the beginning and then turned off when the optimal set-point is satisfied. Therefore, this objective can be realised by

$$
\chi_{i, j}(t+1)-\chi_{i, j}(t) \leq 0, \quad \forall t \in\left[1, n_{s}\right] \subset \mathbb{Z}_{+},
$$

which means that the required parallel pumps can be switched from ON-status to OFFstatus only once.

Thus, the pump scheduling approach in the lower layer can be implemented by solving the following optimisation problem:

\section{Problem 3 (Pump scheduling approach of the WDN).}

$$
\min _{\chi_{i, j}^{*}(t)} \phi_{v}\left\|V_{j}^{p}-V_{j}^{o p t}\right\|_{2}^{2}-\phi_{p} \sum_{i=1}^{n_{c_{j}}} \sum_{t=1}^{H_{l}} \mu_{i} \chi_{i, j}(t),
$$

subject to

$$
\begin{aligned}
& V_{j}^{p}=\sum_{i=1}^{n_{c_{j}}} \sum_{t=1}^{H_{l}} q_{i, j}(t) \Delta t_{l}, \\
& V_{j}^{o p t}=Q_{j}^{o p t} \Delta t_{u}, \\
& q_{i, j}(t)=\chi_{i, j}(t) q_{i, j}^{r}, \\
& \chi_{i+1, j}(t)+\left(1-\chi_{i, j}(t)\right) \leq 1, \\
& \chi_{i, j}(t+1)-\chi_{i, j}(t) \leq 0, \\
& \chi_{i, j}(t)=\{0,1\},
\end{aligned}
$$

where the weight $\phi_{v}$ and $\phi_{p}$ are prioritisation weights, where $\phi_{v}$ should be chosen to be much bigger than $\phi_{p}$ because the main objective is to reach the optimal flow set-point from the upper layer.

By solving Problem 3 for each pumping station, the pump scheduling $\chi_{i, j}^{*}(t)$ for $\forall i \in$ $\left[1, n_{c_{j}}\right] \subset \mathbb{Z}_{+}, \forall j \in\left[1, n_{s}\right] \subset \mathbb{Z}_{+}, \forall t \in\left[1, n_{s}\right] \subset \mathbb{Z}_{+}$can be obtained for the lower layer.

\section{Case study: D-Town water network}

\subsection{Description of D-Town water network}

The D-Town water network shown in Figure 4 is selected as the case study to illustrate the proposed strategy in this paper. The benchmark of D-Town network contains 388 water demand sectors, 405 links (pipes), 7 tanks, which contains multiple unidirectional and bidirectional links. The required pressure for all the water demand sectors is selected to be equal to 20 meters. In order to explore the optimal control strategy, especially for 


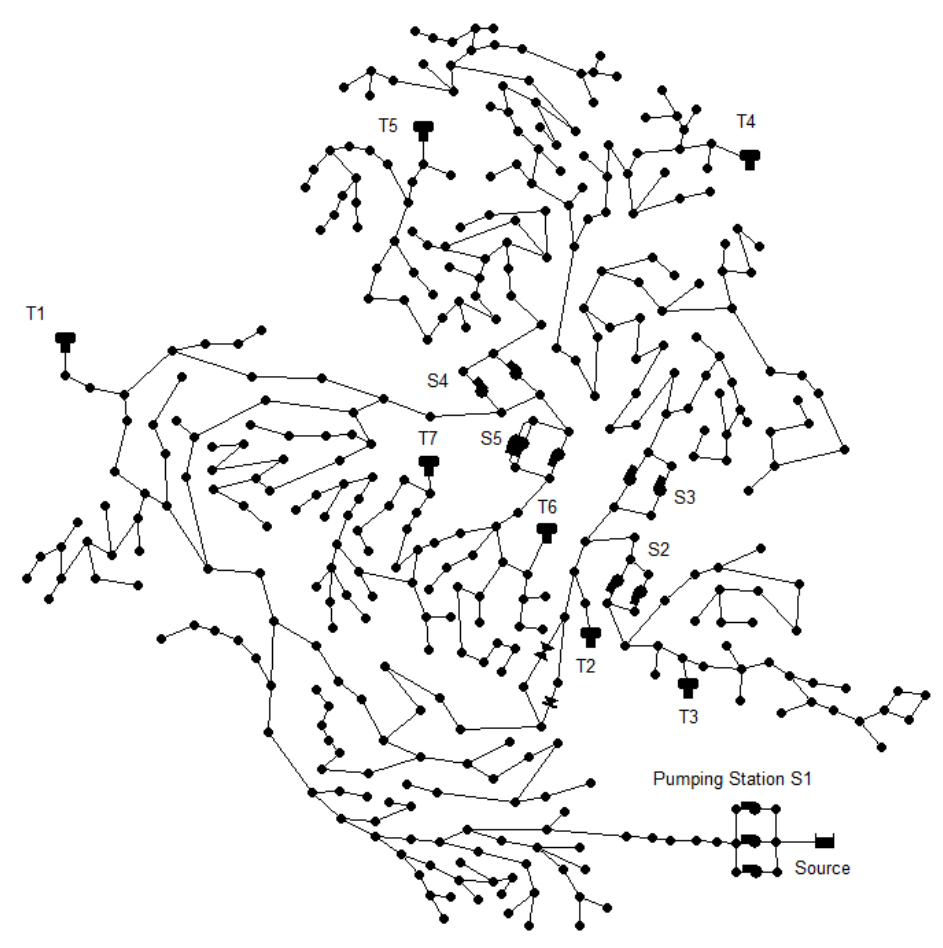

Figure 4: Original topology of the D-Town water network

the NEMPC strategy, the unidirectional pipes and water demand sectors inside can be aggregated into its root node. Therefore, the aggregate topology of the D-Town water network is shown in Figure 5. The required water demands in the root nodes are modified by aggregating the demands from a branch of unidirectional pipes and nodes while the required head of a branch is equivalent to the maximum head in this branch taking the head-loss through the pipes into account as well. Hence, the aggregate model of the D-Town network is selected as the prediction model for the NEMPC strategy and the original one is used in the hydraulic simulator.

The required hydraulic head at each demand node is time-varying during one day since the head-loss through the pipes has been taken into account and the head depends on the water flow. For the control objectives associated to management of this case study, the prioritisations are determined considering the economic objective is the most important and then the safety objective is more significant than the smoothness objective.

As numerous water networks, depending on the scanning time of the telemetry system and on the dynamics of water distribution, a supervision sampling time and control interval of one hour is considered. Furthermore, water consumption in urban areas can be observed with potential pattern on a daily, weekly or monthly basis. In this case study, water consumption is regarded on a daily basis with the proper hourly 24-step-ahead demand forecasting. Under the aforementioned considerations, the MPC prediction horizon of 24 hours is considered appropriate for evaluating the effects of different control strategies on the water network. 


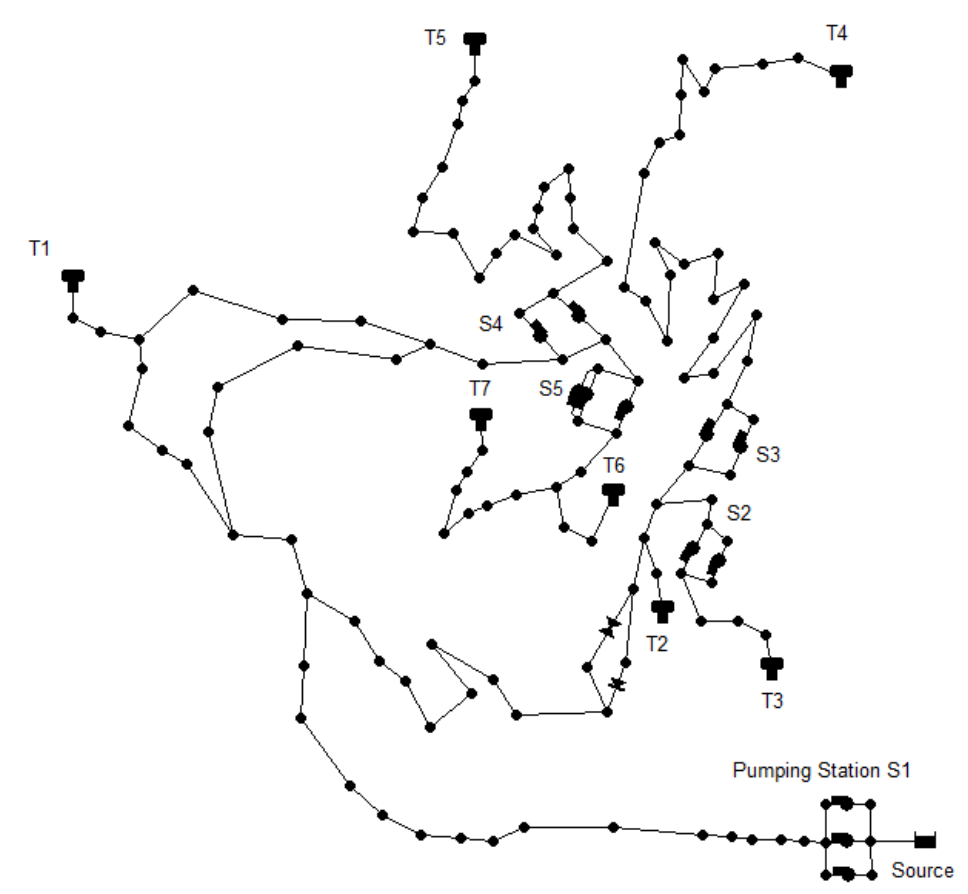

Figure 5: Aggregate topology of the D-Town water network

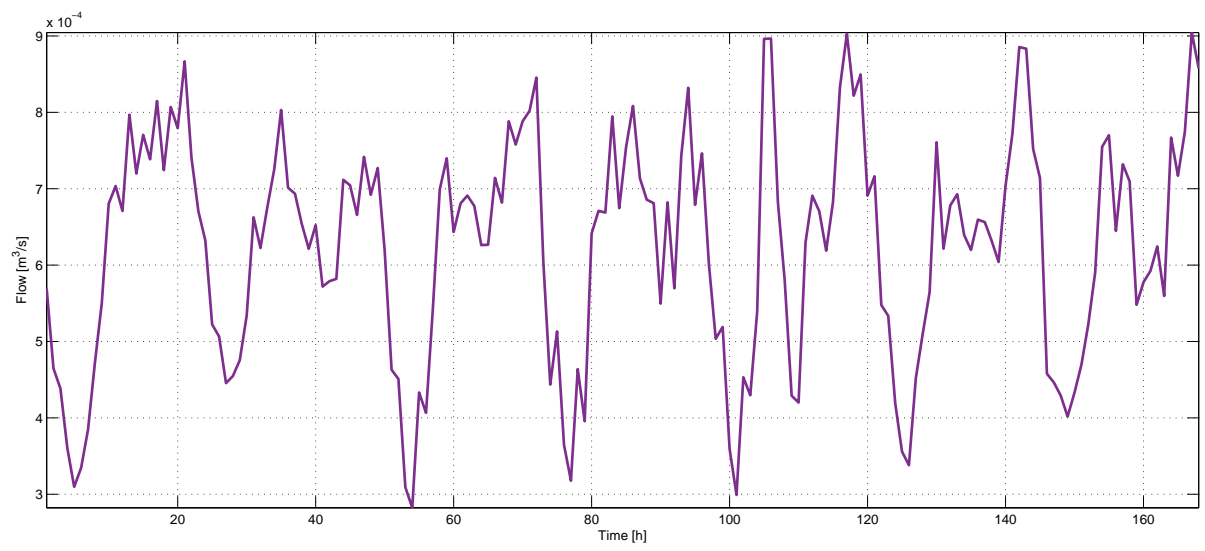

Figure 6: Water demand pattern in the D-Town water network

Moreover, the demand patterns of one week in the database are shown in Figure 6. For the different nodes, the demands has been defined by means of the pattern scaled by a set of numbers. From the control-oriented model, the short-term demand forecasting results are required, which can be obtained by using a certain forecasting method.

\subsection{Validations of the aggregate model of D-Town water network}

It is necessary to verify the behaviour of the aggregate model of the D-Town network is equivalent to the original model of the D-Town network. The validation simulation is 

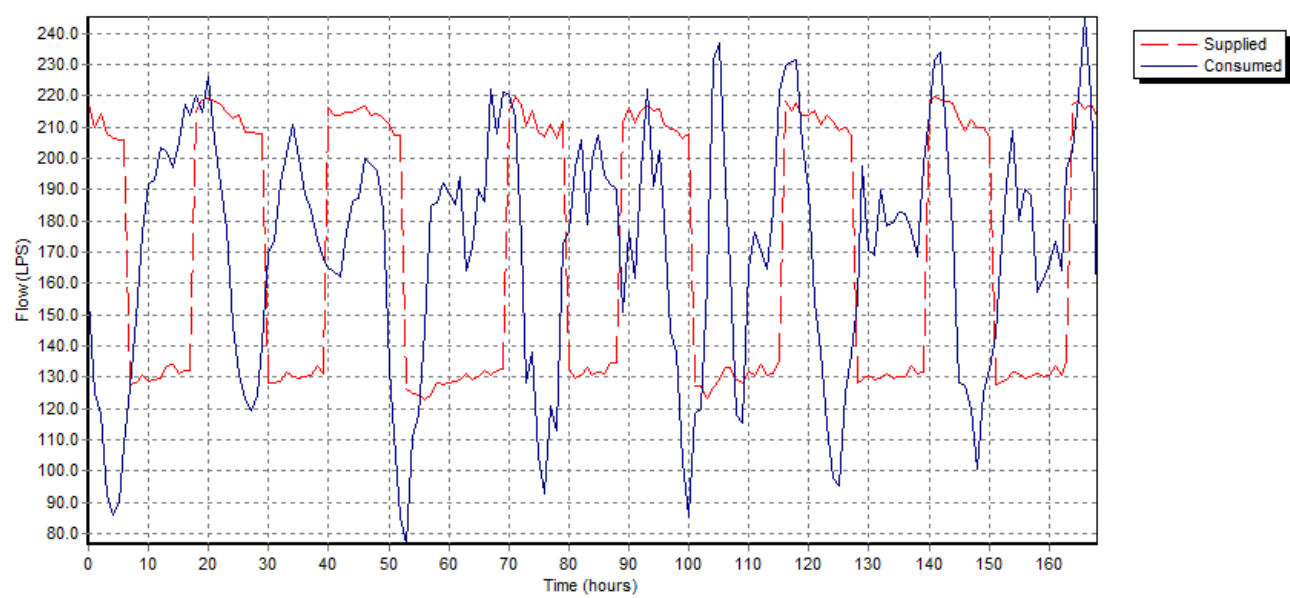

(a) The original D-Town model

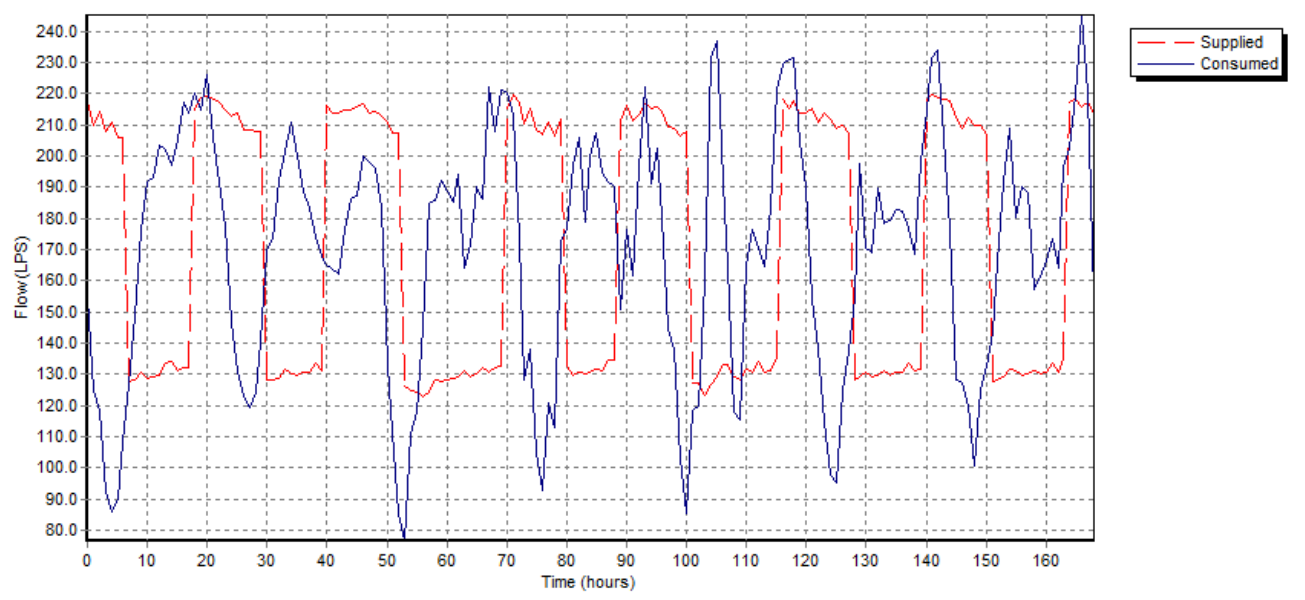

(b) The aggregate D-Town model

Figure 7: Validation of the produced and consumed system flows

realised in the EPANET software. The simple control rules provided in the original network are used for the simulation in four days. As a result, the simulation results with two models are shown in Figure 7(a) and Figure 7(b).

The blue solid lines and red in Figure 7(a) and Figure 7(b) present the total water flow in the network system. It is obvious that results of two figures are very similar. Hence, the aggregate model is proper representation for this case study. For the purpose of simplicity, the aggregate model is used for replacing the original model as the system model in the MPC controller while the original model is used in the simulator. 


\section{Simulation results}

\subsection{On-line simulation platform}

The on-line simulation is carried out in a PC with the CPU of Intel (R) Core (TM) i7-5500U $2.4 \mathrm{GHz}$, the memory of $12 \mathrm{~GB}$ and MATLAB R2014a.The NEMPC strategy is implemented by means of the GAMS ${ }^{2}$ and the CONOPT3 non-linear solver, the EPANET ${ }^{3}$ hydraulic simulator and MATLAB that is used for the communication between the GAMS model of the NEMPC controller and the EPANET hydraulic simulator. Besides, the proposed pump scheduling approach is also implemented in the MATLAB environment. The mixed-integer optimisation problem of the pump scheduling approach is solved by using the MOSEK solver [27]. The topological graph of the communication is shown in Figure 8. The database includes the water demands data and electrical tariff data.

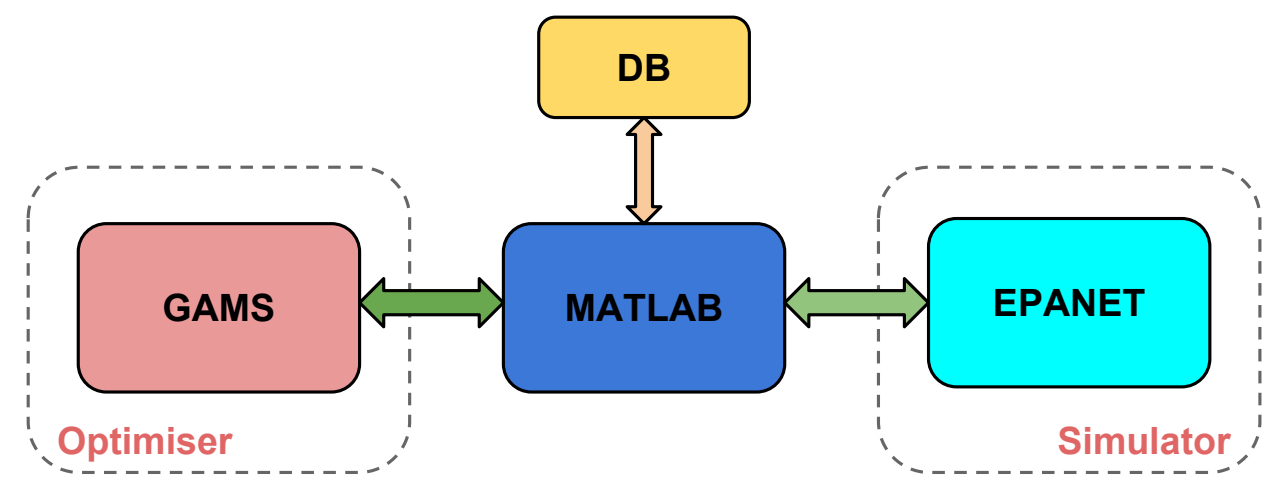

Figure 8: On-line simulation platform

The non-linear optimisation problem is solved using a generalized reduced gradient search [28] implemented in GAMS/CONOPT3 library [29]. It follows an iterative procedure, which can be summarised as follows:

- Initialise and find a feasible solution;

- Compute the Jacobian of the constraints;

- Select a set of basic variables such that the sub-matrix of the basic column from the Jacobian is non-singular;

- Compute the reduced gradient and find a search direction;

- Performing a search in this direction, using a pseudo-Newton process;

\footnotetext{
${ }^{2}$ General Algebraic Modelling System (GAMS) is a programming language for mathematical optimisation and able to solve the complex, large-scale and non-linear optimisation problems [25].

${ }^{3}$ EPANET software is a hydraulic simulator used for the hydraulic behaviour analysis of a WDN. The WDN is built in EPANET consisting of water storage tanks/reservoirs, pumps, valves, pipes and nodes [26].
} 
- Ending the search until a convergence criterion is satisfied.

The detailed description of this algorithm can be found in [29]. This method is well suitable for models with many non-linear constraints.

\subsection{Results}

Problem 1 is implemented in the GAMS programming language. Problem 3 is implemented in the MATLAB environment. The MPC prediction horizon is chosen as 24 with the sampling time of 1 hour in the upper layer. In the lower layer, the computational horizon of the optimisation problem is chosen as 60 with the sampling time of 1 minute. In the upper layer, the prioritisation weights for economics, safety and smoothness objectives are chosen as 10, 1 and 0.1 , respectively. In the lower layer, the prioritisation weights $\phi_{v}$ and $\phi_{p}$ are chosen to be $10: 1$. For the pumping station having three parallel pumps, the weights of $\mu_{1}$, $\mu_{2}$ and $\mu_{3}$ are chosen as 1.5, 1 and 0.5 . For the pumping station having two parallel pumps, the weights of $\mu_{1}$ and $\mu_{2}$ are chosen as 1 and 0.5 . The tolerance of the non-linear solver is set as $10^{-4}$.

The average single-step computation time of solving the upper layer non-linear optimisation problem is 53.21 seconds, being considerably smaller than the sampling time of 1 hour used in this layer. On the other hand, the average single-step computation time of solving the lower layer mixed-integer optimization problem is 4.19 seconds, being also smaller than the sampling time of 1 minute used in this layer. Thus, the proposed strategy can be applied in real-time.

Figure 9 shows the head evolutions of selected storage tanks. The dash blue line denotes the optimal hydraulic heads of the storage tanks. It is obvious that the head has daily quasiperiodic feature mainly because of the daily water demands and electricity tariffs. Moreover, results from the EPANET hydraulic simulator are plotted in the cyan lines. By means of this state comparison between the EPANET simulator and optimiser, it is clear that the optimal system trajectories can be reached with the two-layer control strategy.

The selected average hourly water flows of the pumping stations are shown in the Figure 10 in the magenta lines. The average hourly water flow of a pumping station can be calculated by

$$
Q_{j}^{\text {ave }}=\frac{\sum_{i=1}^{N_{q}} q_{i, j}^{\text {real }}}{60} .
$$

The water flow in Figure 10(a) is associated to the pumping station S1 and the average water flow is approximately similar and sometimes below the optimal flow set-point because the actual pumping flow is varying during each control interval depending on the boundary heads. Furthermore, the patterns of electrical tariff are added for reference in all the plots in Figure 10. In general, the optimal flows are small when the electricity price is expensive. On the contrary, the flows are increasing when the electricity price becomes cheaper.

Figure 11 shows the optimal flow set-point and actual flow evolution of the valve in D-Town water network. The type of the valve is flow-controlled. The simulation flow is approximately tracking the optimal flow set-point. Hence, there is single-layer control for 


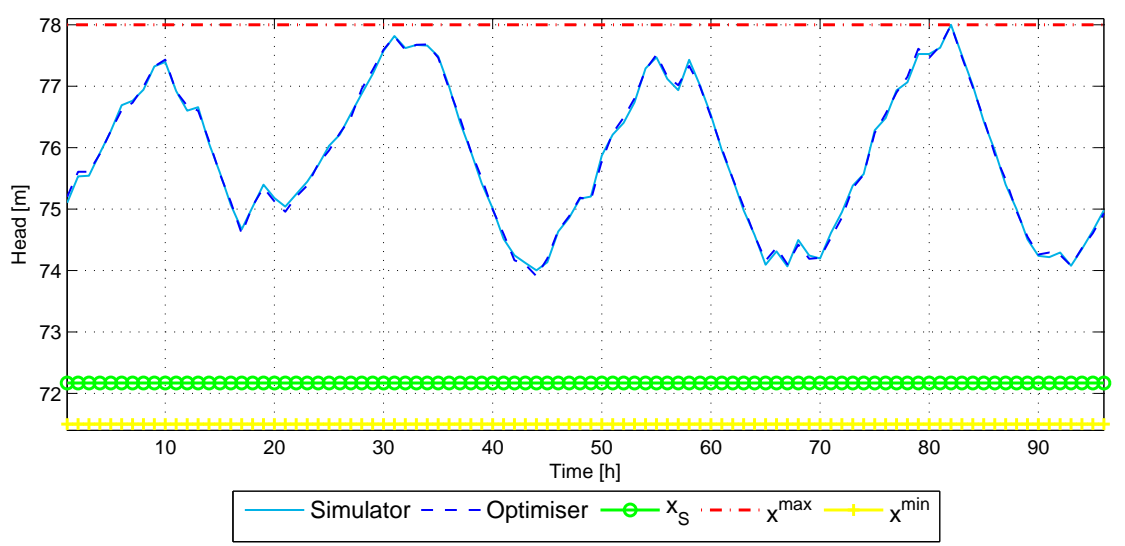

(a) Tank: T1

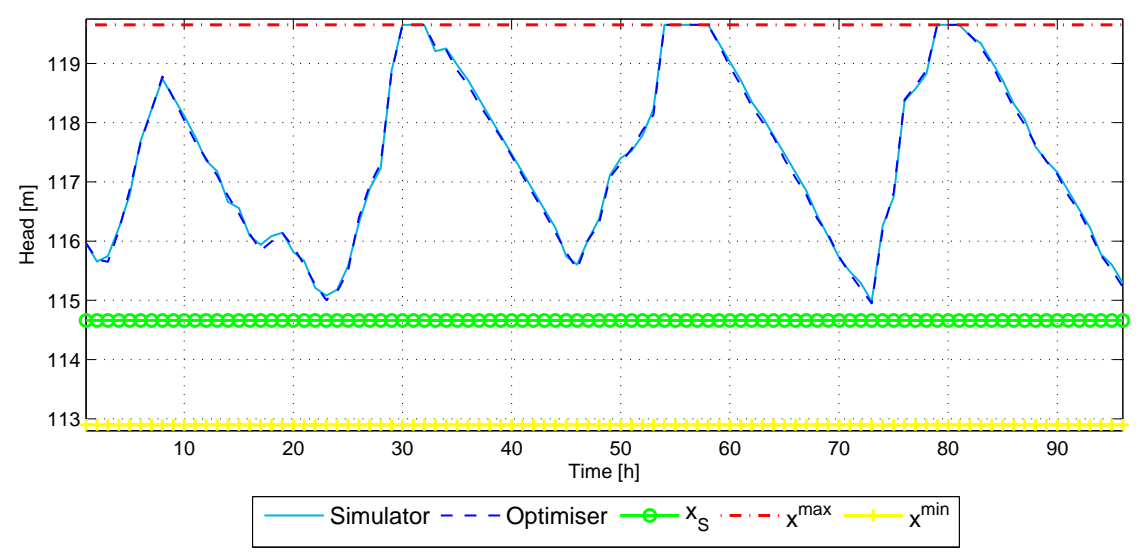

(b) Tank: T3

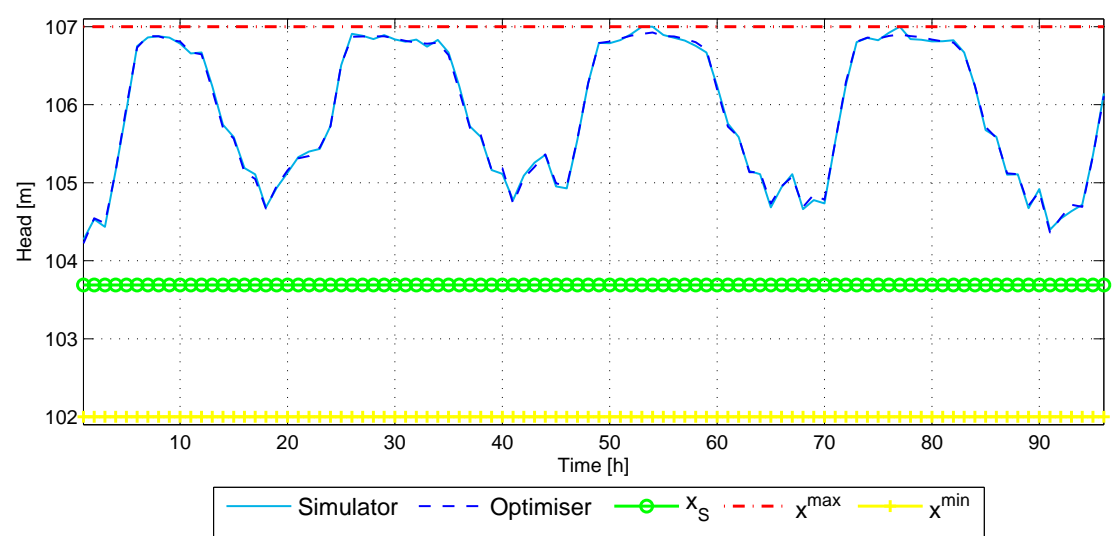

(c) Tank: T7

Figure 9: Results of the head evolutions of storage tanks 


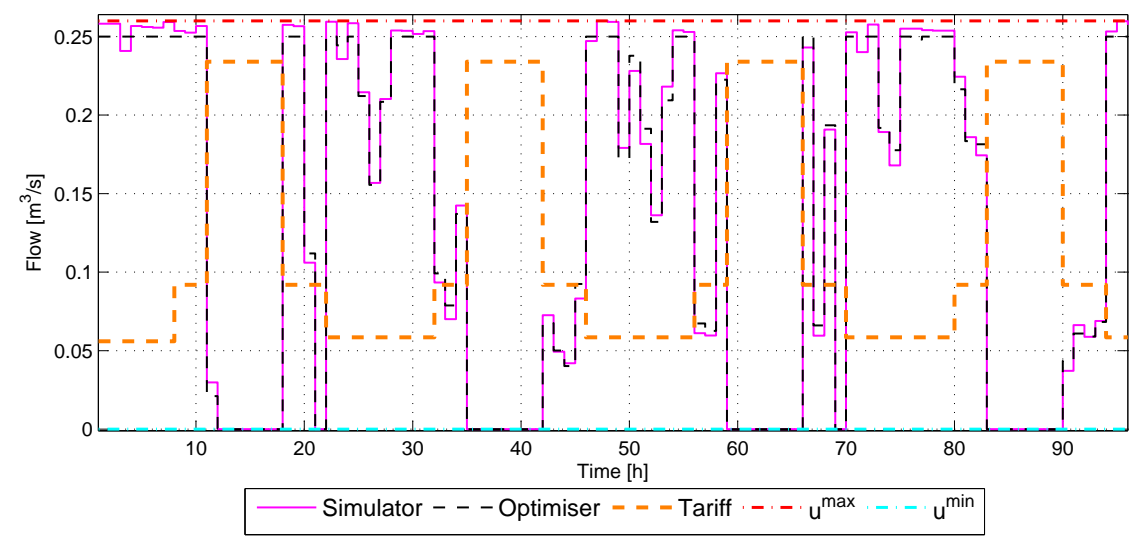

(a) Pumping station: S1

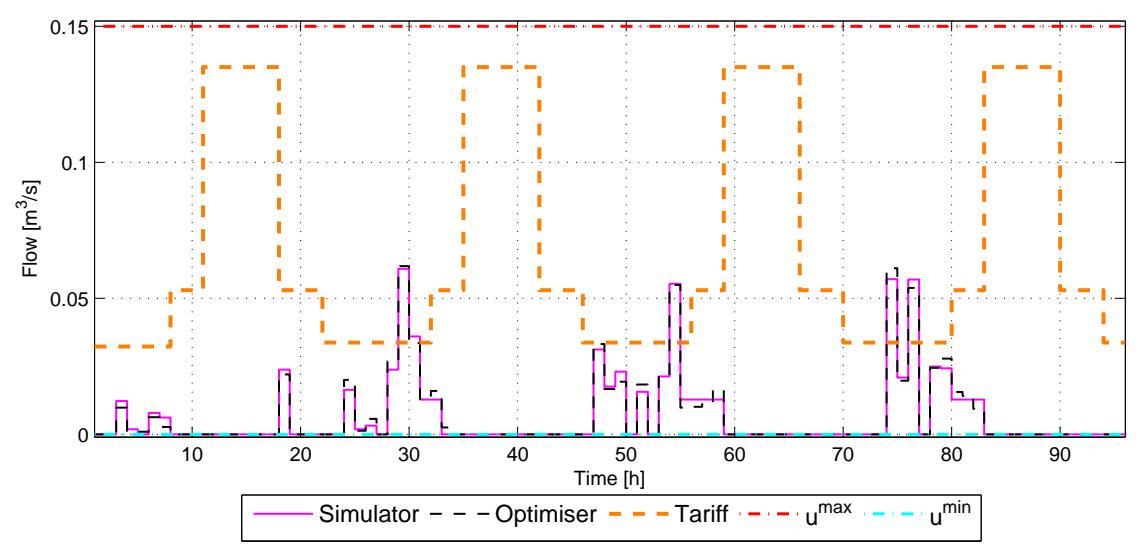

(b) Pumping station: S2

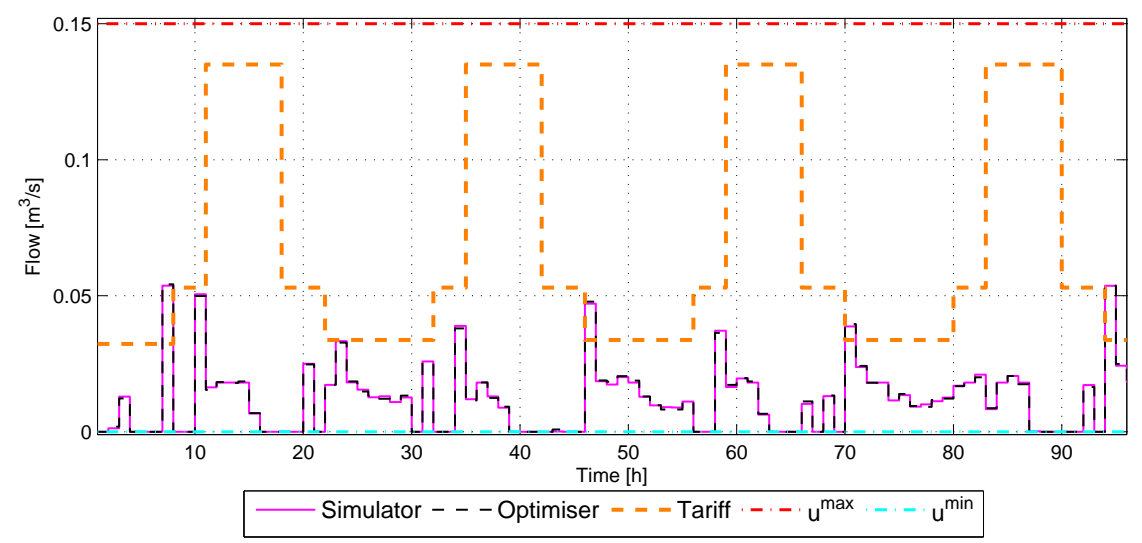

(c) Pumping station: S4

Figure 10: Results of the flows through pumping stations 


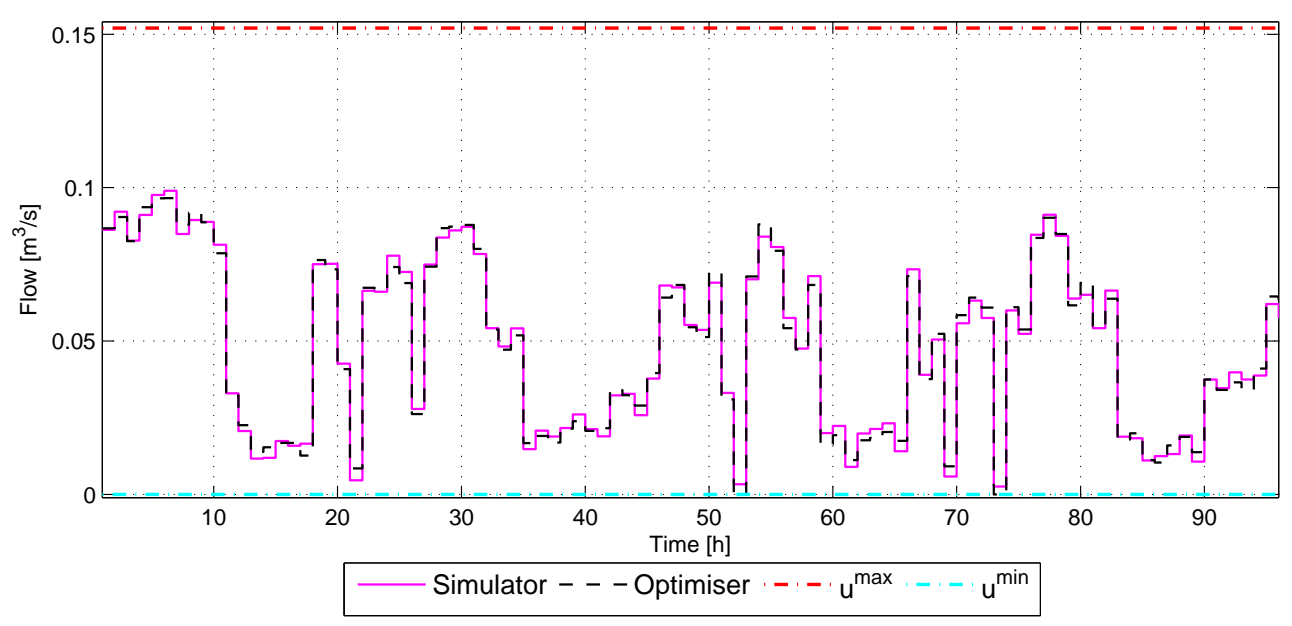

Figure 11: Result of the flow through the valve: V2

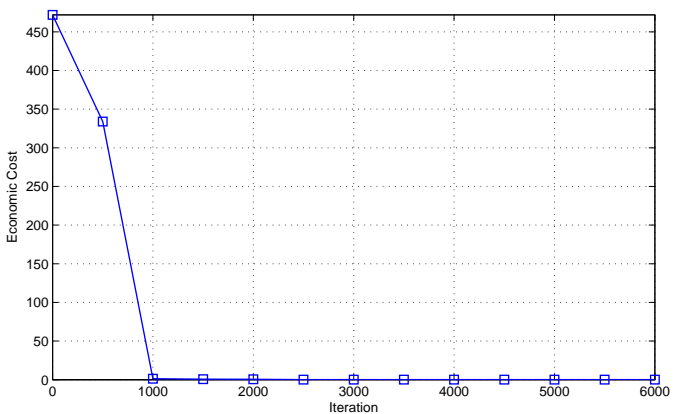

(a) EMPC cost evaluated at each iteration

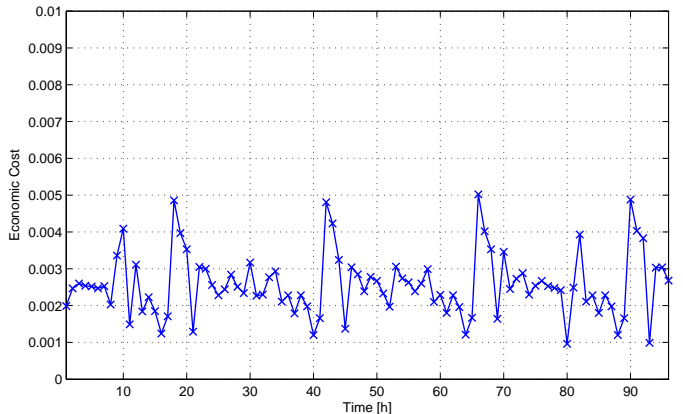

(b) EMPC cost with EPANET as virtual reality

Figure 12: Economic costs in NEMPC

the valve and the optimal flow set-point is applied to the valve during one hour at a MPC step from the upper layer. But from this plot, there are small differences between the actual flow and optimal flow set-point.

Figure 12(a) presents the economic cost achieved by the EMPC controller at each sampling time (EMPC cost). It can be observed, that after a transient, the cost converges to a stable small cost. These results are confirmed with the results presented in Figure 12(b) where the EMPC cost obtained using the EPANET simulator to emulate the real network is presented. From this last figure, it can be observed that the EMPC cost converges to a stable mean value. The cost fluctuations around this mean value are due to the mismatch between the control-oriented model used by the EMPC and the high fidelity hydraulic cost used by EPANET. 
Table 2: Safety heads of storage tanks

\begin{tabular}{cc}
\hline Tank & Safety head $(m)$ \\
\hline T1 & 72.25 \\
T2 & 65.42 \\
T3 & 114.38 \\
T4 & 133.90 \\
T5 & 106.41 \\
T6 & 102.83 \\
T7 & 102.97 \\
\hline
\end{tabular}
by using the successive linear programming (SLP) in [30] and approximately $168,118 €$ by using the pseudo-genetic algorithm (PGA) in [31], the two-layer NEMPC strategy is able to bring less operational costs for the management of the D-Town water network.

\section{Conclusions}

In this paper, a two-layer NEMPC control strategy of WDNs is proposed. The optimal set-points for actuators have been calculated by means of solving an non-linear optimisation problem and subsequently used for deploying the pump scheduling approach considering the 
ON/OFF manner of the pumps. Throughout the case study of the D-Town water network, he on-line simulation results are shown the feasibility of the proposed control strategy and its economic merits. Compared with previous MPC strategies of the WDN only considering the flow-based model, the NEMPC strategy of the WDN is able to meet all the demands in water demand sectors with their required pressure at the same time. Hence, the NEMPC strategy is considered an adequate control strategy for the operational management of the WDN. Furthermore, the lower layer determines the hourly flow set-points for each pumps in the pumping stations. For pumps, a logic controller should be used. The difficulty of the lower layer is to realise the optimal flow of the pumps within an accurate range. On the other hand, it is necessary to maximise the service life of the pump by minimising the switches, which will be considered in the pumping scheduling approach as the further research.

As the on-line simulation platform presented in this paper, the EPANET hydraulic simulator is used, which has a number of the physical constraints for the pumping stations, such as the pumping curve, speed and efficiency curve. Therefore, the head evolutions in storage tanks in the EPANET hydraulic simulator is tracking the optimal set-points provided by the two-layer control strategy, which means the proposed control strategy is effective.

A large class of state-of-the-art methods for solving large-scale non-linear mixed integer optimisation problems, such as branch-and-bound and evolutionary-programming methods cannot provide a guarantee of convergence to an optimum in a limited computation time. The main advantage behind using this two-layer approach is to take advantage of the guaranteed convergence properties of non-linear programming, to obtain a reference optimal control strategy with a computation time that is well within the real-time limitations. The second layer translates the reference control strategy into ON/OFF schedules for specific pumps, which can be implemented directly in the actuators, as shown in this work, using a realistic network simulator. The simulation results show that NEMPC in the upper layer as the reference strategy are followed closely by means of the lower layer scheme.

\section{Acknowledgement}

This work has been partially supported by the European Commission through the EFFINET project (ref. FP7-ICT-2012-318556), by the MEICOMP through the project CICYT ECOCIS (ref. DPI2013-48243-C2-1-R) and by MEICOMP and FEDER through the project CICYT HARCRICS (ref. DPI2014-58104-R). The authors also thank M. Urrea and J. Romera for providing technical supports.

\section{References}

[1] D. Mayne, Model predictive control: Recent developments and future promise, Automatica 50 (12) (2014) 2967-2986.

[2] S. Qin, T. Badgwell, A survey of industrial model predictive control technology, Control Engineering Practice 11 (7) (2003) 733-764.

[3] M. Heidarinejad, J. Liu, P. Christofides, Economic model predictive control of nonlinear process systems using Lyapunov techniques, AIChE Journal 58 (3) (2012) 855-870.

[4] M. Ellis, P. Christofides, On finite-time and infinite-time cost improvement of economic model predictive control for nonlinear systems, Automatica 50 (10) (2014) 2561-2569. 
[5] M. Ellis, P. Christofides, Integrating dynamic economic optimization and model predictive control for optimal operation of nonlinear process systems, Control Engineering Practice 22 (2014) 242-251.

[6] D. Savić, G. Walters, Genetic Algorithms for Least-Cost Design of Water Distribution Networks, Journal of Water Resources Planning and Management 123 (2) (1997) 67-77.

[7] G. Cembrano, G. Wells, J. Quevedo, R. Pérez, R. Argelaguet, Optimal control of a water distribution network in a supervisory control system, Control Engineering Practice 8 (10) (2000) 1177-1188.

[8] C. Ocampo-Martinez, V. Puig, G. Cembrano, J. Quevedo, Application of MPC strategies to the management of complex networks of the urban water cycle, IEEE Control Systems Magazine 33 (1) (2013) $15-41$.

[9] J. Grosso, C. Ocampo-Martinez, V. Puig, B. Joseph, Chance-constrained model predictive control for drinking water networks, Journal of Process Control 24 (5) (2014) 504-516.

[10] Y. Wang, C. Ocampo-Martinez, V. Puig, Robust model predictive control based on Gaussian Processes: Application to drinking water networks, in: European Control Conference (ECC), Linz, Austria, 3292$3297,2015$.

[11] J. Marques, M. Cunha, D. Savić, Multi-objective optimization of water distribution systems based on a real options approach, Environmental Modelling \& Software 63 (2015) 1-13.

[12] G. Sankar, S. Kumar, S. Narasimhan, S. Narasimhan, S. Bhallamudi, Optimal control of water distribution networks with storage facilities, Journal of Process Control 32 (2015) 127-137.

[13] Y. Wang, C. Ocampo-Martinez, V. Puig, Stochastic Model Predictive Control based on Gaussian Processes applied to Drinking Water Networks, IET Control Theory \& Applications 10 (8) (2016) 947-955.

[14] Y. Wang, V. Puig, G. Cembrano, Economic MPC with Periodic Terminal Constraints of Nonlinear Differential-Algebraic-Equation Systems: Application to Drinking Water Networks, in: European Control Conference (ECC), Aalborg, Denmark, 1013-1018, 2016.

[15] Y. Wang, T. Alamo, V. Puig, G. Cembrano, Periodic Economic Model Predictive Control with Nonlinear-Constraint Relaxation for Water Distribution Networks, in: IEEE Conference on Control Applications (CCA), Buenos Aires, Argentina, 1167-1172, 2016.

[16] H. Saadeldin, Pressure, Leakage and Energy Management in Water Distribution Systems, Ph.D. thesis, De Montfort University, 2011.

[17] C. Sun, V. Puig, G. Cembrano, Combining CSP and MPC for the operational control of water networks, Engineering Applications of Artificial Intelligence 49 (2016) 126-140.

[18] J. Quevedo, J. Saludes, V. Puig, J. Blanch, Short-term demand forecasting for real-time operational control of the Barcelona water transport network, in: 22nd Mediterranean Conference on Control and Automation (MED), Palermo, Italy, 990-995, 2014.

[19] Y. Wang, C. Ocampo-Martinez, V. Puig, J. Quevedo, Gaussian-process-based demand forecasting for predictive control of drinking water networks, in: Critical Information Infrastructures Security, Springer, 69-80, 2016.

[20] L. Grüne, J. Pannek, Nonlinear Model Predictive Control: Theory and Algorithms, Communications and Control Engineering, Springer, 1st edn., 2011.

[21] H. Han, J. Qiao, Nonlinear Model-Predictive Control for Industrial Processes: An Application to Wastewater Treatment Process, IEEE Transactions on Industrial Electronics 61 (4) (2014) 1970-1982.

[22] M. Brdys, B. Ulanicki, Operational Control of Water Systems: Structures, Algorithms and Applications, Upper Saddle River, Prentice-Hall, 1994.

[23] G. Cembrano, J. Quevedo, V. Puig, R. Pérez, J. Figueras, J. M. Verdejo, I. Escaler, G. Ramón, G. Barnet, P. Rodríguez, M. Casas, PLIO: a generic tool for real-time operational predictive optimal control of water networks, Water Science and Technology 64 (2) (2011) 448-459.

[24] M. Lopez, T. Prasad, B. Paechter, Ant Colony Optimization for Optimal Control of Pumps in Water Distribution Networks, Journal of Water Resources Planning and Management 134 (4) (2008) 337-346.

[25] R. Rosenthal, GAMS - A User's Guide, GAMS Development Corporation, URL http://www.gams. com/help/topic/gams.doc/userguides/GAMSUsersGuide.pdf, 2013.

[26] L. Rossman, Epanet 2.0 Users Manual, URL https://www.epa.gov/water-research/epanet, 2000. 
[27] MOSEK ApS, The MOSEK optimization toolbox for MATLAB manual. Version 7.1 (Revision 28)., URL http://docs.mosek.com/7.1/toolbox/index.html, 2015.

[28] G. Gabriele, K. Ragsdell, The Generalized Reduced Gradient Method: A Reliable Tool for Optimal Design, ASME. J. Eng. Ind. 99 (2) (1977) 394-400.

[29] GAMS, The Solver Manuals, GAMS Development Corporation, URL http://www.gams.com/help/ topic/gams.doc/solvers/allsolvers.pdf, 2016.

[30] E. Price, A. Ostfeld, Battle of Background Leakage Assessment for Water Networks Using Successive Linear Programing, in: 16th Water Distribution System Analysis Conference (WDSA), vol. 89, Bari, Italy, 45-52, 2014.

610 [31] P. Iglesias-Rey, F. Martínez-Solano, D. M. Meliá, P. Martínez-Solano, BBLAWN: A Combined Use of Best Management Practices and an Optimization Model Based on a Pseudo-Genetic Algorithm, in: 16th Water Distribution System Analysis Conference (WDSA, vol. 89, Bari, Italy, 29-36, 2014. 